\title{
Assessment of female breast dose for thoracic cone-beam CT using MOSFET dosimeters
}

\author{
Wenzhao Sun ${ }^{1, *}$, Bin Wang ${ }^{1, *}$, Bo Qiu ${ }^{1}$, Jian Liang ${ }^{1}$, Weihao $X^{1}{ }^{1}$, Xiaowu Deng ${ }^{1}$, \\ Zhenyu Qi ${ }^{1}$ \\ ${ }^{1}$ Department of Radiation Oncology, Sun Yat-Sen University Cancer Center, Collaborative Innovation Center for Cancer \\ Medicine, State Key Laboratory of Oncology in South China, Guangzhou 510060, China \\ *These authors have contributed equally to this work \\ Correspondence to: Zhenyu Qi, email: qizhy@sysucc.org.cn \\ Keywords: radiotherapy, cone beam computed tomography (CBCT), metal oxide semiconductor field-effect transistor (MOSFET), \\ breast dose, image registration \\ Received: July 26, $2016 \quad$ Accepted: January 23, $2017 \quad$ Published: February 21, 2017
}

ABSTRACT

Objective: To assess the breast dose during a routine thoracic cone-beam CT ( $С В C T)$ check with the efforts to explore the possible dose reduction strategy.

Materials and Methods: Metal oxide semiconductor field-effect transistor (MOSFET) dosimeters were used to measure breast surface doses during a thorax kV CBCT scan in an anthropomorphic phantom. Breast doses for different scanning protocols and breast sizes were compared. Dose reduction was attempted by using partial arC CBCT scan with bowtie filter. The impact of this dose reduction strategy on image registration accuracy was investigated.

Results: The average breast surface doses were $20.02 \mathrm{mGy}$ and $11.65 \mathrm{mGy}$ for thoracic СВCT without filtration and with filtration, respectively. This indicates a dose reduction of $41.8 \%$ by use of bowtie filter. It was found $220^{\circ}$ partial arc scanning significantly reduced the dose to contralateral breast $(44.4 \%$ lower than ipsilateral breast), while the image registration accuracy was not compromised.

Conclusions: Breast dose reduction can be achieved by using ipsilateral $220^{\circ}$ partial arc scan with bowtie filter. This strategy also provides sufficient image quality for thorax image registration in daily patient positioning verification.

\section{BACKGROUND}

The therapeutic goal of advanced radiotherapy techniques like intensity modulated radiation therapy (IMRT) has focused on the precise irradiation of target volume while sparing normal tissues and organs as much as possible. This has created the need for more accurate patient positioning, especially for the treatment of thoracic cancer. Various image-guided devices like on-board kilovoltage cone beam computed tomography (CBCT) have thus been increasingly implemented in IMRT practice $[1,2]$. By taking CBCT images on a daily or weekly basis, the possible patient's setup error can be detected and subsequently adjusted prior to the treatment $[3,4]$. Several pilot studies also explore the feasibility of fast dose calculation based on CBCT images, so as to perform adaptive radiotherapy to further improve the treatment geometrical accuracy [5-7]. The rapidly growing use of $\mathrm{CBCT}$, however, raises the issue of additional imaging dose and concomitant increases in secondary cancer risk [8-10]. There is a general consensus that low dose protocols should be preferred whenever possible to reduce the imaging dose and volume of exposed anatomy [11]. This is particularly true for female breast during a thoracic CBCT for lung cancer, which is often inadvertently irradiated even though it is not the imaged tissue of interest.

Several investigations have revealed the absorbed breast dose from single $\mathrm{kV}$-CBCT ranges from several mGy to a few tens of $\mathrm{mGy}$, depending on different imaging equipments and scanning protocols [11-13]. The stochastic health risk as a result of thoracic CBCT is not trivial, considering that for every 0.1 Gy of low dose irradiation at the age of 20 in female patients, the 
life time attributable risk of developing breast cancer increases $0.4 \%$ [14]. In fact, the female breast is more radiosensitive than previously assumed, according to the newly modified tissue weighting factors. The International Commission on Radiological Protection (ICRP) has recently changed its value from 0.05 to 0.12 for the breast tissue [15].

To better estimate the health effects from imaging exposures, it is essential to determine individual organ doses associated with various imaging procedures during a radiotherapy episode. Conventionally, these point doses were often measured in an anthropomorphic phantom using $\mathrm{LiF}$ thermoluminescent dosimeters (TLD) $[11,13]$. The major advantages of LiF TLDs are their small size and tissue equivalent response. But they are also known to be tedious to use. The fact that acquiring moderately accurate, reproducible results using TLDs requires a stringent pre-calibration, annealing and post-irradiation readout process hinders their use in the diagnostic radiology clinic. Recently, the utilization of metal oxide semiconductor field effect transistor (MOSFET) technology in CT dosimetry has begun to appear in many peer-reviewed radiology publications [16-19]. Compared with TLDs, the MOSFET dosimeters can offer a fast, simple and inexpensive means to conduct a point dose measurement in radiation beams.

While the CBCT dose to the female breast has been previously investigated [11-13], the possible dose reduction strategies are less reported. A newly published paper discussed one feasible way to reduce additional dose to the patient due to CBCT by decreasing the $\mathrm{mAs}$ per frame and the number of projections per CBCT and concluded that image registration can be successfully performed even for lowest possible settings [20]. However, this pilot study only explored the effect of total mAs on image registration and there maybe have some other solutions such as partial arc scanning. Also direct dose measurement may better assess the breast dose during CBCT than the CT dose index (CTDI) used. In this study, we will focus on the imaging exposure to the healthy breast in daily thoracic CBCT for lung cancer patient positioning. The absorbed dose to the breast was measured with MOSFET dosimeters in a femaleconfigured anthropomorphic phantom. Different scanning protocols were selected and the effects on image quality were analyzed. Proper strategies have been developed to reduce exposure in $\mathrm{CBCT}$ without reducing the image information on the registration process.

\section{RESULTS}

\section{Calibration of the MOSFET dosimeter}

The derived MOSFET sensitivity was $15.77 \pm 0.14$ $\mathrm{mV} / \mathrm{cGy}$ and $14.88 \pm 0.11 \mathrm{mV} / \mathrm{cGy}$ for blank field and filtrated field, respectively (Table 1). The average reading of repeated measurements for the typical thoracic CBCT (protocol B, no filtration) was $27.23 \mathrm{mV} \pm 0.73 \mathrm{mV}$, resulting in a measurement reproducibility of $2.7 \%$ at the tested dose level.

\section{Female breast dose of $\mathrm{kV}$ CBCT by ART phantom}

No significant dependence was found between the breast surface dose and the breast volume (Table 2). The average breast surface doses of various breast volumes were $20.02 \mathrm{mGy}$ and $11.65 \mathrm{mGy}$ for bank field and filtrated field, respectively, indicating a dose reduction of $41.8 \%$ by use of bowtie filter.

The doses of bilateral breasts measured by MOSFET dosimeters were shown in Table 3. It was observed that partial arc scanning produced a relatively lower breast dose than full arc mode. Particularly, the use of ipsilateral $220^{\circ}$ partial arc significantly reduced the contralateral breast doses by about $16 \%$ and $44.4 \%$ for bank field and filtrated field, respectively, compared to the ipsilateral breast doses.

\section{Evaluation of CBCT image quality}

For S20 collimator setting, both uniformity and LCV were improved by using bowtie filter (Table 4). As for M20 collimator setting, the bowtie filter improved the LCV while no improvements were seen on image uniformity.

\section{Registration accuracy}

As shown in Table 5, image registration can be successfully performed for both CBCT protocols. It was noted that the systematic setup and registration errors were eliminated from the registration results. The maximum difference in registration between two protocols was observed to be within $0.1 \mathrm{~cm}$.

\section{DISCUSSION}

The MOSFET dosimeters have been recommended by many authors for diagnostic CT dosimetry [16-19]. As the reproducibility of MOSFET dosimeters was closely correlated with the applied dose [21], the CMRP MOSFETs were calibrated at the dose range of a normal thorax CBCT, yielding a measurement accuracy within $3 \%$. The derived MOSFET sensitivity was 14.88 and $15.77 \mathrm{mV} / \mathrm{cGy}$ for measurement with and without bowtie filter, respectively. This indicated a minimal sensitivity variation within $6 \%$ for blank and filtrated beam qualities, which is consistent with the previous finding [22]. The possible reason is that the filtration changes the energy spectrum and the high atomic number material 
Table 1: Results and parameters for MOSFET calibration

\begin{tabular}{|c|c|c|c|c|c|c|c|}
\hline $\begin{array}{l}\text { Peak Voltage } \\
(\mathrm{kV})\end{array}$ & $\begin{array}{c}\text { Scanning } \\
\text { configuration }\end{array}$ & $\begin{array}{c}\text { HVL }^{\text {a }}(\mathbf{m m} \\
\text { Al) }\end{array}$ & $\begin{array}{c}\text { Effective } \\
\text { Energy } \\
(\mathrm{keV})\end{array}$ & $\left(\mu_{\mathrm{en}} / \rho\right)_{\mathrm{w}, \mathrm{air}}$ & $\mathbf{B}_{\mathrm{w}}$ & $\mathbf{P}_{\text {stem }}$ & $\begin{array}{r}\text { Sensitivity } \\
(\mathrm{mV} / \mathrm{cGy})\end{array}$ \\
\hline 120 & $\mathrm{~F} 0+\mathrm{S} 20$ & 8.1 & 56.5 & 1.043 & 1.502 & 1 & 15.77 \\
\hline 120 & $\mathrm{~F} 1+\mathrm{S} 20$ & 9.0 & 60.0 & 1.047 & 1.509 & 1 & 14.88 \\
\hline
\end{tabular}

${ }^{\text {a }}$ Half value layer.

Table 2: Breast surface doses of different breast volumes for thoracic CBCT scans (A, B, C, and D corresponds to the breast volume of $200,400,600$, and 1200 cc respectively)

\begin{tabular}{|c|c|c|c|c|}
\hline Filtration & $\begin{array}{c}A \\
(200 \mathrm{cc})\end{array}$ & $\begin{array}{c}\text { B } \\
(400 \mathrm{cc})\end{array}$ & $\begin{array}{c}C \\
(600 \mathrm{cc})\end{array}$ & $\begin{array}{c}D \\
(1200 \text { cc })\end{array}$ \\
\hline F0 & $19.44 \pm 1.05$ & $20.29 \pm 0.00$ & $19.44 \pm 1.05$ & $20.92 \pm 0.00$ \\
\hline F1 & $12.17 \pm 0.00$ & $12.17 \pm 0.00$ & $11.33 \pm 0.00$ & $10.91 \pm 0.00$ \\
\hline
\end{tabular}

Protocol B with or without filter was used. The breast dose was presented in the form of $\mathrm{D}_{\text {mean }} \pm \mathrm{SD} \mathrm{mGy}$.

Table 3: Comparison of radiation doses delivered to ipsilateral breast vs. contralateral breast for full arc and partial arc CBCT

\begin{tabular}{lccccc}
\hline & \multicolumn{2}{c}{ Protocol B } & & \multicolumn{2}{c}{ Protocol C } \\
\cline { 2 - 3 } \cline { 5 - 6 } Filtration & Ipsilateral breast & Contralateral breast & & Ipsilateral breast & Contralateral breast \\
\hline F0 & $19.44 \pm 1.05$ & $19.44 \pm 1.05$ & & $17.97 \pm 0.40$ & $15.43 \pm 1.43$ \\
F1 & $12.17 \pm 0.00$ & $12.17 \pm 0.00$ & & $11.33 \pm 0.00$ & $6.30 \pm 0.69$ \\
\hline
\end{tabular}

The breast doses were measured using ART phantom with $200 \mathrm{cc}$ artificial breast. The measured dose was presented in the form of $\mathrm{D}_{\text {mean }} \pm \mathrm{SD} \mathrm{mGy}$.

Table 4: Results of uniformity and LCV tests for thoracic CBCT images

\begin{tabular}{lcccc}
\hline & $\begin{array}{c}\text { Full Arc } \\
\text { (F0M20) }\end{array}$ & $\begin{array}{c}\text { Full Arc } \\
\text { (F1M20) }\end{array}$ & $\begin{array}{c}\text { Full Arc } \\
\text { (F0S20) }\end{array}$ & $\begin{array}{c}\text { Full Arc } \\
\text { (F1S20) }\end{array}$ \\
\hline Non-uniformity(\%) & 2.38 & 5.16 & 2.77 & 1.09 \\
LCV $(\%)$ & 1.16 & 1.11 & 1.24 & 0.64 \\
\hline
\end{tabular}

Table 5: Comparison of registration results between full arc CBCT and partial arc CBCT for thoracic cancer patient positioning verification

\begin{tabular}{|c|c|c|c|c|c|c|}
\hline \multirow{2}{*}{$\begin{array}{l}\text { Nominal shifted } \\
\text { distance } \\
\text { (cm) }\end{array}$} & \multicolumn{3}{|c|}{ Protocol B (full arc) } & \multicolumn{3}{|c|}{ Protocol C (partial arc) } \\
\hline & LAT & LONG & VERT & LAT & LONG & VERT \\
\hline Normal setup (0) & 0.04 & 0.14 & 0.04 & 0.06 & 0.16 & 0.06 \\
\hline Anterior $1 \mathrm{~cm}$ & 0.03 & -0.11 & -1.05 & 0.03 & -0.05 & -1.07 \\
\hline Superior $1 \mathrm{~cm}$ & -0.01 & -0.96 & 0.00 & -0.02 & -0.97 & -0.01 \\
\hline Left $1 \mathrm{~cm}$ & -0.96 & -0.04 & 0.00 & -0.96 & -0.12 & 0.00 \\
\hline
\end{tabular}

The systematic setup and registration error was subtracted from the registration results. 
Table 6: Scanning parameters for thorax CBCT. (Protocol A was used for MOSFET calibration, protocol B was used for nominal thorax CBCT scan, and protocol $\mathrm{C}$ was used for partial-arc scan investigation.)

\begin{tabular}{|c|c|c|c|}
\hline & Protocol A & Protocol B & Protocol C \\
\hline Tube voltage $[\mathrm{kV}]$ & 120 & 120 & 120 \\
\hline Nonminal $\mathrm{mA} /$ frame & 25 & 25 & 25 \\
\hline Nominal mS/frame & 40 & 40 & 40 \\
\hline Frames & 650 & 650 & 397 \\
\hline Total mAs & 650 & 650 & 397 \\
\hline $\mathrm{FOV}[\mathrm{mm}]$ & S & M & M \\
\hline Collimator & 20 & 20 & 20 \\
\hline Filter & $\mathrm{F} 0 / \mathrm{F} 1$ & $\mathrm{~F} 0 / \mathrm{F} 1$ & $\mathrm{~F} 0 / \mathrm{F} 1$ \\
\hline Gantry Rotation & $\begin{array}{c}\text { stationary } \mathrm{X} \text {-ray tube } \\
\left(0^{\circ}\right)\end{array}$ & $\begin{array}{l}\text { rotational x-ray tube } \\
\qquad\left(-180^{\circ} \sim 180^{\circ}\right)\end{array}$ & $\begin{array}{l}\text { rotational x-ray tube } \\
\qquad\left(-110^{\circ} \sim 110^{\circ}\right)\end{array}$ \\
\hline
\end{tabular}

in the sensitive volume of the MOSFET (silicon oxide) tends to over respond to the low energy $\mathrm{x}$-rays due to photoelectric absorption effects. In this study, calibration was carried out individually for blank field and filtrated field for the purpose of accuracy.

The entrance surface dose was often used as an indicator of patient's exposure in radiographic applications. This is due to the fact that the $\mathrm{kV}$ radiation dose is greatest at the skin surface as well as the surface dose assessment is practical feasible. As shown by our results, the breast surface doses derived from a thorax $\mathrm{CBCT}$, measured with MOSFET dosimeters, were on average $20.02 \mathrm{mGy}$. Although the observed imaging dose is small compared with the radiotherapy dose, the potential risk for stochastic effects could not be neglected considering the total dose from all imaging sessions during intensive IGRT is considerable. Kan et al [11] estimated that patient position verification using $\mathrm{kV} \mathrm{CBCT}$ (Varian, $\mathrm{OBI}$ ) on a daily basis (35 fractions) could cause 1.5 to 2.0 Gy extra dose to some critical organs, which might increase the overall risk of the secondary cancer by $3 \%$ to $4 \%$. Considering XVI usually produces larger imaging dose than OBI [13], more attention should be paid to justification for use of XVI system.

Several efforts to reduce patient's exposure during CBCT were reported [20, 23-25]. These investigations were performed by minimizing the total $\mathrm{mAs}[20,23]$ and the use of bowtie filter [24,25]. Besides these, partial arc CBCT has recently be demonstrated to be a good supplement to current dose reduction strategies for patient positioning verification of head \& neck and pelvis [25]. In this study, our results approved partial arc scan could also be used for thoracic cancer patient positioning by selecting proper scanning parameters. It was seen the use of $220^{\circ}$ partial arc scan effectively reduced the radiation to the female breasts, while the image registration accuracy was not compromised. The observed dose reduction by partial arc may be due to the fact that $220^{\circ}$ partial arc applied less number of projections and thus decreasing the total mAs. More importantly, ipsilateral $220^{\circ}$ partial arc setting can minimize radiation exposures to the contralateral breast. This may be particularly suitable for routine CBCT in some cases such as breast cancer, in which sparing the heathy breast is one of major concerns.

The use of bowtie filter has several advantages, including a lower skin dose, reduction of the image saturation of the $\mathrm{kV}$ detector panel, and elimination of the cupping artifacts across the FOV [26]. As shown by our results, the introduction of bowtie filter produced significantly lower dose to the breasts compared to the none filtrated field, which was in consistent with previously published studies [24, 25]. In addition, CBCT acquisition using bowtie filter and S20 collimator produce much better image quality in terms of image uniformity and LCV, compared to F0 and S20 combination. The fact that $\mathrm{CBCT}$ acquisition with bowtie filter and M20 collimator yield relatively larger non-uniformity than F0 and M20 combination (5.16\% vs $2.38 \%$ ) may result from the off-axial $\mathrm{kV}$ field used by M20 setup. Consequently, the bowtie filter has been recommended as a routine setting for thoracic $\mathrm{CBCT}$ by our institutional protocol.

\section{CONCLUSIONS}

The breast dose due to thoracic CBCT can be significantly reduced by using ipsilateral $220^{\circ}$ partial arc scan with bowtie filter. This strategy also provides sufficient image quality for thorax image registration in daily patient positioning verification. 


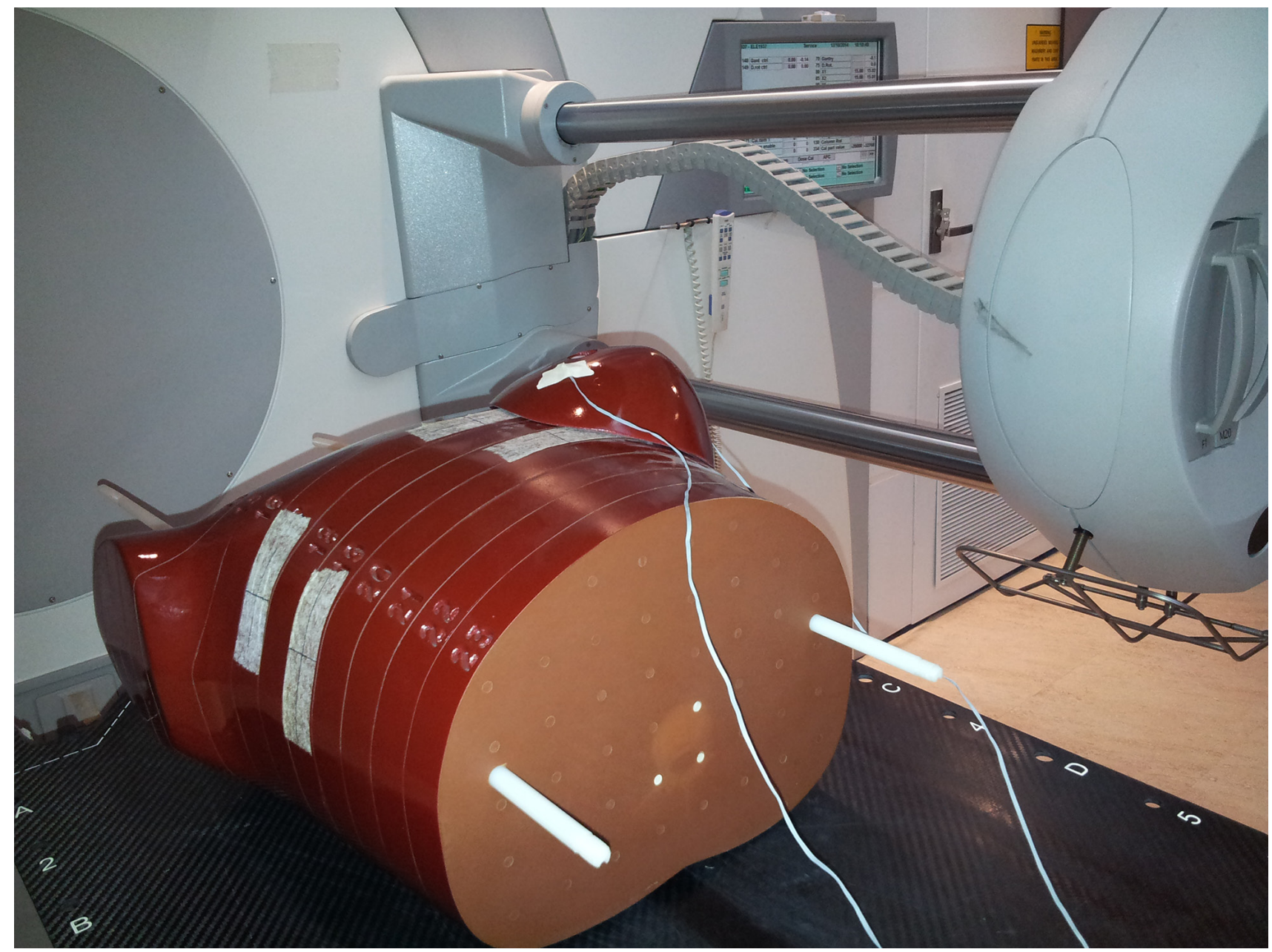

Figure 1: Setup of breast CBCT dose measurement using a female-configured anthropomorphic phantom and MOSFET detectors.
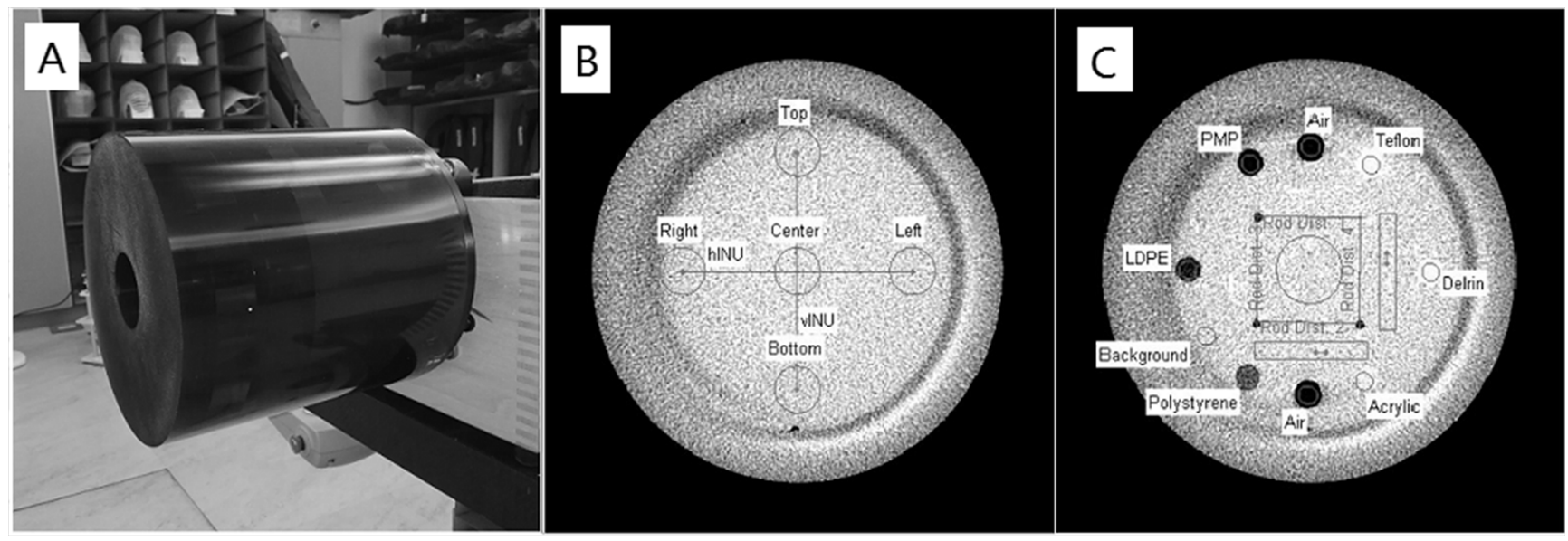

Figure 2: (A) Catphan 503 phantom, (B) images of 486 module used for image uniformity test and (C) 404 module used for low contrast visibility evaluation. 


\section{MATERIALS AND METHODS}

\section{kV CBCT}

Experiments were performed on the X-ray Volume Imager (XVI) mounted onto an Elekta Synergy linear accelerator (Elekta, Crawley, UK). The XVI system can provide different combinations of $\mathrm{kV}$ collimators (labeled as 10 and 20 which can generate the axial field length of about $135 \mathrm{~mm}$ and $143 \mathrm{~mm}$ at the isocenter, respectively), field of views (FOV, labeled as S, M and L for small FOV (270 $\mathrm{mm}$ in diameter), medium FOV (410 $\mathrm{mm}$ in diameter) and large FOV (500 $\mathrm{mm}$ in diameter), respectively) and filtration cassettes (labeled as F0 and F1 for blank filter and bowtie filter, respectively). For a typical thorax CBCT scan, the medium FOV with collimator 20 (i.e. M20) was recommended by the manufacturer. However, the small FOV with collimator 20 (i.e. S20) was preferred in the calibration process as the central axis of the $\mathrm{kV}$ radiation is in line with the center of the $\mathrm{kV}$ detector panel in the small FOV configuration and offset in the M20 configuration. Also, the X-ray tube was stationed at $0^{\circ}$ position rather than rotation during the calibration for the purpose of accuracy. The detailed scan parameters for MOSFET dosimeter calibration and thorax CBCT scan were given in Table 6. The acquired image data was processed by using the software XVI release 3.5 (Elekta, Crawley, UK).

\section{MOSFET dosimetry system}

The p-channel MOSFETs (brand named "MOSkin") together with a portable reader were supplied by the Center of Medical Radiation Physics (CMRP), University of Wollongong, Australia. The physical performance of this dosimeter was well documented in the literature for external beam radiotherapy $[27,28]$, interventional radiology procedures [22] and diagnostic radiology dosimetry $[16,17]$.

The MOSFET dosimeter is connected to the CMRP reader system when it is used. The reader contains circuits not only for tracking and logging the threshold voltage but also for applying gate bias as required during exposure. A positive gate bias of $5 \mathrm{~V}$ was selected during irradiation to increase the dosimeter's sensitivity and linearity [29]. Possible signal drifts that occurred in time during and after irradiation have been corrected by using deconvolution methods in this dosimetry system [30]. The stated uncertainty associated with this reader was $\pm 1 \mathrm{mV}$ in the integral mode and was found to be lower than $\pm 2 \mathrm{mV}$ before and after the irradiation in the real-time mode [31].

Prior to use, the MOSFET dosimeter was calibrated against a $0.6 \mathrm{cc}$ ion chamber (TW30013, PTW, German) using the X-ray source from the Elekta XVI system at $120 \mathrm{kV}$. As adding the filtration would change the X-ray spectrum which may vary the MOSFET sensitivity [22], the calibration was conducted for F0 and F1 filter, respectively. The half value layers (HVL) of the primary $\mathrm{X}$-ray beam at different conditions were determined by using the ion chamber.

During the calibration, the MOSFET dosimeters were placed on the surface of a $40 \times 40 \times 20 \mathrm{~cm}^{3}$ solid water slab phantom at the iso-center with their sensitive region facing the x-ray beam. The CBCT scanning parameters used for calibration were listed in Table 6. The delivered dose $\left(D_{w, z=0}\right)$ was obtained with the ion chamber at the iso-center using the in-air method following the recommendation of AAPM TG-61 report [32]:

$$
\mathrm{D}_{\mathrm{w}, \mathrm{z}=0}=\mathrm{MN}_{\mathrm{K}} \mathrm{B}_{\mathrm{w}} \mathrm{P}_{\text {stem, air }}\left[\left(\frac{\bar{\mu}_{\mathrm{en}}}{\rho}\right)_{\text {air }}^{\mathrm{w}}\right]_{\text {air }}
$$

where $\mathrm{M}$ is the ion chamber reading corrected for temperature, pressure, ion recombination and polarity effect. $\mathrm{N}_{\mathrm{K}}$ indicates the air-kerma calibration factor relevant to the beam quality. $\mathrm{B}_{\mathrm{w}}$ indicates the backscatter factor. $\mathrm{P}_{\text {stem, air }}$ indicates the chamber stem correction factor.

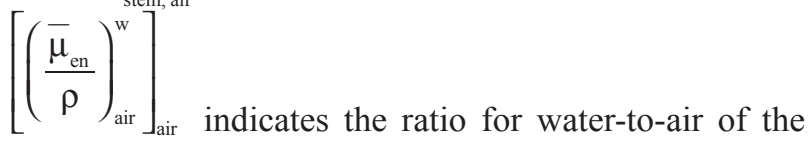
mean mass energy absorption coefficients averaged over the incident photon spectrum. The associated calibration parameters were detailed in Table 1.

All the measurements were repeated five times and the average readings were used to calculate the MOSFET sensitivity:

$$
\text { Sensitivity }(m V / c G y)=\Delta \operatorname{VTH}(m V) / \mathrm{D}_{\mathrm{w}, z=0}(c G y)
$$

where $\triangle \mathrm{VTH}$ is the threshold voltage shift of the MOSFET dosimeter before and after the irradiation.

To evaluate the measurement reproducibility (S), the MOSFETs were scanned with a typical thoracic CBCT protocol (protocol B, see Table 1) using the calibration setup. Measurements were repeated ten times and the reproducibility of the MOSFETs were computed as:

$$
\mathrm{S}=\frac{1}{\bar{M}} \times \sqrt{\frac{\sum_{\mathrm{i}=1}^{\mathrm{n}}\left(\overline{\mathrm{M}}-\mathrm{M}_{\mathrm{i}}\right)^{2}}{\mathrm{n}-1}} \times 100 \%
$$

where $\bar{M}$ is the average MOSFET reading for ten equal and consecutive irradiations $(\mathrm{n}=10)$.

\section{Breast dose measurement}

As shown in Figure 1, the breast doses were measured with MOSFET dosimeters positioned at 3, 6, 9, 12 o'clock and at the center on breast surface in a female-configured anthropomorphic phantom (The Alderson Radiation Therapy phantom, Radiology Support Devices, Inc. USA). To fully 
assess the effectiveness of partial arc CBCT scanning, both ipsilateral and contralateral breast were investigated in this experiment. Measurements were performed with artificial breasts of different sizes to determine the influence of breast volume on breast dose. The possible effects of filtration and varying rotation were also tested.

\section{Analysis of image quality}

The image uniformity and low contrast visibility were investigated in a Catphan 503 phantom (The Phantom Laboratory, Salem, NY, USA) (Figure 2A) to evaluate the impact of dose reduction on CBCT image quality.

\section{Uniformity}

The phantom's homogeneous module CTP 486 was imaged (Figure 2B). Five $1 \mathrm{~cm}$ diameter circular regions of interest (ROIs) were selected on the middle slice of the images at the module center and at 3, 6, 9, and 12 o'clock positions. All the peripheral ROIs were carefully set to be within the "inner circle" (uniformity module) of the image, which was $2 \mathrm{~cm}$ from the edge of the image area. The mean CT value of each ROI was then determined, by means of which the maximum and minimum $\mathrm{CT}$ number was identified. The non-uniformity index was calculated using the formula:

$$
\text { Non }- \text { uniformity }=\left(\frac{\text { mean }(\text { high })-\text { mean }(\text { low })}{\text { mean }(\text { high })}\right)
$$

\section{Low contrast visibility}

To estimate the low contrast visibility, the module CTP 404 was scanned (Figure 2C). Two circular ROIs with a diameter of $0.35 \mathrm{~cm}$ were identified within the lowdensity polyethylene (LDPE) and polystyrene (PS) inserts, respectively, on the middle layer of the images. The mean pixel values within these ROIs and the associated standard deviations (SD) were recorded. The low contrast visibility was calculated as follows:

$$
\text { low contrast visibility } \%=\frac{\left(\mathrm{CT}_{\text {polystyrene }}-\mathrm{CT}_{\mathrm{LDPE}}\right) / 10}{\left\{\frac{\left(\mathrm{mean}_{\text {polystyrene }}-\mathrm{mean}_{\mathrm{LDPE}}\right)}{\left(\mathrm{SD}_{\text {polystyrene }}-\mathrm{SD}_{\mathrm{LDPE}}\right) / 2}\right\}}
$$

\section{Registration accuracy}

The female-configured anthropomorphic phantom in Figure 1 was used to analyze the registration accuracy for different CBCT scanning protocols. The phantom was firstly scanned by a spiral CT at $120 \mathrm{kVp}$ with $3 \mathrm{~mm}$ slice thickness and $3 \mathrm{~mm}$ spacing. The CT images were transferred to the XVI workstation as reference images.

Keeping the same phantom setup, CBCT acquisitions were performed with M20 collimator cassette and F1 filter using varying rotations (i.e., $360^{\circ}$ full arc scanning vs. $220^{\circ}$ partial arc scanning). The systematic setup and registration error was hence determined by image registration of CT and CBCT images using Elekta Synergy R3.5 software. In this study, the grayscale match algorithm was preferred and the registration box was selected to cover the whole chest area according to the departmental IGRT protocols. The phantom was then shifted by $1 \mathrm{~cm}$ in three directions to simulate the patient's inter-fractionation motion. New CBCT images were acquired and the detected positioning error was compared with the nominal shifted distance.

\section{ACKNOWLEDGMENTS}

This work was supported by grants: National Natural Science Foundation of China, No.81371710, Science and Technology Program of Guangdong Province, China, No.2013B021800149, Science and Technology Program of Guangzhou, China, No.201607010199.

\section{CONFLICTS OF INTEREST}

The authors declare no conflicts of interest.

\section{REFERENCES}

1. Moore CJ, Amer A, Marchant T, Sykes JR, Davies J, Stratford J, McCarthy C, MacBain C, Henry A, Price P. Developments in and experience of kilovoltage X-ray cone beam image-guided radiotherapy. The British journal of radiology. 2006; 79:S66-78.

2. Oldham M, Létourneau D, Watt L, Hugo G, Yan D, Lockman D, Kim LH, Chen PY, Martinez A, Wong JW. Cone-beam-CT guided radiation therapy: A model for on-line application. Radiotherapy and oncology. 2005; 75:271. E271-271. E278.

3. Borst GR, Sonke JJ, Betgen A, Remeijer P, Van Herk M, Lebesque JV. Kilo-voltage cone-beam computed tomography setup measurements for lung cancer patients; first clinical results and comparison with electronic portal-imaging device. International Journal of Radiation Oncology Biology Physics. 2007; 68:555-561.

4. Bissonnette JP, Purdie TG, Higgins JA, Li W, Bezjak A. Cone-beam computed tomographic image guidance for lung cancer radiation therapy. International Journal of Radiation Oncology Biology Physics. 2009; 73:927-934.

5. Nijkamp J, Pos FJ, Nuver TT, De Jong R, Remeijer P, Sonke JJ, Lebesque JV. Adaptive radiotherapy for prostate cancer using kilovoltage cone-beam computed tomography: 
first clinical results. International Journal of Radiation Oncology Biology Physics. 2008; 70:75-82.

6. Richter A, Hu Q, Steglich D, Baier K, Wilbert J, Guckenberger M, Flentje M. Investigation of the usability of conebeam CT data sets for dose calculation. Radiation Oncology. 2008; 3:1.

7. Srinivasan K, Mohammadi M, Shepherd J. Cone beam computed tomography for adaptive radiotherapy treatment planning. Journal of Medical and Biological Engineering. 2014; 34:377-385.

8. Quinn A, Holloway L, Koh ES, Delaney G, Arumugam S, Goozee G, Metcalfe P. Radiation dose and contralateral breast cancer risk associated with megavoltage cone-beam computed tomographic image verification in breast radiation therapy. Practical radiation oncology. 2013; 3:93-100.

9. Donovan E, James H, Bonora M, Yarnold J, Evans P. Second cancer incidence risk estimates using BEIR VII models for standard and complex external beam radiotherapy for early breast cancer. Medical physics. 2012; 39:5814-5824.

10. Kim DW, Chung WK, Yoon M. Imaging doses and secondary cancer risk from kilovoltage cone-beam CT in radiation therapy. Health physics. 2013; 104:499-503.

11. Kan MW, Leung LH, Wong W, Lam N. Radiation dose from cone beam computed tomography for image-guided radiation therapy. Int J Radiat Oncol Biol Phys. 2008; 70:272-279.

12. Zhang Y, Wu H, Chen Z, Knisely JP, Nath R, Feng Z, Bao $\mathrm{S}$, Deng J. Concomitant Imaging Dose and Cancer Risk in Image Guided Thoracic Radiation Therapy. Int J Radiat Oncol Biol Phys. 2015; 93:523-531.

13. Shah A, Aird E, Shekhdar J. Contribution to normal tissue dose from concomitant radiation for two common $\mathrm{kV}-\mathrm{CBCT}$ systems and one MVCT system used in radiotherapy. Radiother Oncol. 2012; 105:139-144.

14. Royal HD. Effects of low level radiation-what's new? Semin Nucl Med. 2008; 38:392-402.

15. The 2007 Recommendations of the International Commission on Radiological Protection. ICRP publication 103. Ann ICRP. 2007; 37:1-332.

16. Lian CPL, Young A, Cutajar D, Freeman N, Rosenfeld AB. Organ point dose measurements in clinical multi slice computed tomography (MSCT) examinations with the MOSkin radiation dosimeter. Radiation Measurements. 2013; 55: 56-59.

17. Lian CPL, Wong J, Young A, Cutajar D, Petasecca M, Lerch $\mathrm{M}$, Rosenfeld AB. Measurement of multi-slice computed tomography dose profile with the Dose Magnifying Glass and the MOSkin radiation dosimeter. Radiation Measurements. 2012; 55:51-55.

18. Ding GX, Coffey CW. Dosimetric evaluation of the OneDose $^{\mathrm{TM}}$ MOSFET for measuring kilovoltage imaging dose from image-guided radiotherapy procedures. Med Phys. 2010; 37:4880-4885.
19. Kumar AS, Singh IR, Sharma SD, Ravindran BP. Performance characteristics of mobile MOSFET dosimeter for kilovoltage X-rays used in image guided radiotherapy. J Med Phys. 2015; 40:123-128.

20. Loutfi-Krauss B, Kohn J, Blumer N, Freundl K, Koch T, Kara E, Scherf C, Rodel C, Ramm U, Licher J. Effect of dose reduction on image registration and image quality for cone-beam CT in radiotherapy. Strahlenther Onkol. 2015; 191:192-200.

21. Cheung T, Yu PKN, Butson MJ. Low-dose measurement with a MOSFET in high-energy radiotherapy applications. Radiation Measurements. 2005; 39:91-94.

22. Safari MJ, Wong JH, Ng KH, Jong WL, Cutajar DL, Rosenfeld AB. Characterization of a MOSkin detector for in vivo skin dose measurements during interventional radiology procedures. Med Phys. 2015; 42:2550-2558.

23. Wang J, Li T, Liang Z, Xing L. Dose reduction for kilovotage cone-beam computed tomography in radiation therapy. Phys Med Biol. 2008; 53:2897-2909.

24. Ding GX, Munro P. Radiation exposure to patients from image guidance procedures and techniques to reduce the imaging dose. Radiother Oncol. 2013; 108:91-98.

25. Ding GX, Munro P, Pawlowski J, Malcolm A, Coffey CW. Reducing radiation exposure to patients from $\mathrm{kV}-\mathrm{CBCT}$ imaging. Radiother Oncol. 2010; 97:585-592.

26. Page 64, XVI R5.0 Instructions for use for Elekta Synergy ${ }^{\mathrm{R}}$, Elekta Axesse $^{\mathrm{TM}}$, Elekta Infinity ${ }^{\mathrm{TM}}$, Versa $\mathrm{HD}^{\mathrm{TM}}$.

27. Kwan IS, Rosenfeld AB, Qi ZY, Wilkinson D, Lerch ML, Cutajar DL, Safavi-Naeni M, Butson M, Bucci J, Chin Y. Skin dosimetry with new MOSFET detectors. Radiation measurements. 2008; 43:929-932.

28. Qi ZY, Deng XW, Huang SM, Zhang L, He ZC, Li XA, Kwan I, Lerch M, Cutajar D, Metcalfe P. In vivo verification of superficial dose for head and neck treatments using intensity-modulated techniques. Medical physics. 2009; 36:59-70.

29. Kaplan GI, Rosenfeld AB, Allen BJ, Booth JT, Carolan MG, Holmes-Siedle A. Improved spatial resolution by MOSFET dosimetry of an x-ray microbeam. Med Phys. 2000; 27 : 239-244.

30. Rosenfeld AB, Lerch ML, Kron T, Brauer-Krisch E, Bravin A, Holmes-Siedle A, Allen BJ. Feasibility study of online high-spatial-resolution MOSFET dosimetry in static and pulsed x-ray radiation fields. IEEE Transactions on Nuclear Science. 2001; 48:2061-2068.

31. Zilio VO, Joneja OP, Popowski Y, Rosenfeld A, Chawla R. Absolute depth-dose-rate measurements for an ${ }^{192}$ Ir HDR brachytherapy source in water using MOSFET detectors. Med Phys. 2006; 33: 1532-1539.

32. Ma CM, Coffey CW, DeWerd LA, Liu C, Nath R, Seltzer SM, Seuntjens JP, American Association of Physicists in M. AAPM protocol for 40-300 kV x-ray beam dosimetry in radiotherapy and radiobiology. Med Phys. 2001; 28:868-893. 